\title{
Tumour cell dissemination and growth of metastasis
}

\author{
Christoph A. Klein
}

I am grateful for the letter of S. Koscielny and $\mathrm{M}$. Tubiana because it allows me to clarify a few points. In the Opinion article (Parallel progression of primary tumours and metastases. Nature Rev. Cancer $\mathbf{9}$, 302-312 (2009)) $)^{1}$ I opposed two models of cancer progression: parallel progression, suggesting early dissemination of tumour cells from the primary tumour and ectopic selective adaptation (which is associated with the emergence of genomes fully able to form metastases); and linear progression, which places major evolutionary events in the primary tumour and late dissemination of fully malignant cells that subsequently grow to manifest metastases.

For reasons not explained in their letter the authors declare parallel progression inconsistent with clinical data. This conclusion is derived from their mathematical model of cancer progression. They assume disseminating cancer cells to be autonomously growing and suggest that the primary tumour at metastasis initiation is only slightly smaller than at the time of tumour diagnosis ${ }^{2,3}$. If correct, metastases grow much faster than primary tumours. However, growth rates and proliferation indices of paired metastases and primary tumours are similar, with metastases growing up to twice as fast as primary tumours ${ }^{1}$. The marginal increase of growth rate has prompted most authors who modelled cancer progression on clinical data to conclude that dissemination of tumour cells occurs long before the diagnosis of the primary tumour and that metastatic colonies and the primary tumour grow in parallel over prolonged periods $s^{4-6}$.

More important are ex vivo analyses of disseminated tumour cells (DTCs). Mathematical models of cancer progression are imprecise for the difference between dissemination of tumour cells and the initiation of metastatic growth. Clinical data sets do not report when tumour cells disseminate, but they document the diagnosis of metastasis. Therefore, one can only assess the link between tumour size and the probability of metastasis; the latency period and the nature of this link need to be explored. Genomic analyses of DTCs provided evidence that ectopically residing tumour cells have strikingly different genotypes to the predominant clone of the primary tumour at the time of surgery and DTC sampling ${ }^{7-10}$. If metastasis founder cells (MFCs) accumulated genomic changes in the primary site and disseminated shortly before surgery, as suggested by Koscielny and Tubiana, it is unclear why this difference would be observed. By contrast, high-resolution molecular analysis showed that some DTCs resemble an early stage of genomic progression shown by the primary tumour, and longitudinal studies revealed ongoing genomic evolution of DTCs - presumably outside the primary tumour - until the manifestation of metastasis ${ }^{11}$. Finally, dissemination of tumour cells from the earliest stages of the primary tumour is now documented in breast and other cancers with a minor increase of dissemination in larger tumours ${ }^{12,13}$. Evidently, the link between tumour size and clinically manifest metastasis is not dissemination but colonization.

Finally, the authors declare that the parallel progression model is incompatible with the cancer stem cell hypothesis. As defined above, parallel progression does not address this issue. Genetic heterogeneity of DTCs has repeatedly been documented $^{8-10,14}$, and heterogeneity is also expected for epigenetic states. Therefore, productive and non-productive DTCs are likely to coexist. However, evidence suggests that specific mechanisms are active during cancer initiation ${ }^{15}$ that might select for the early dissemination of cancer stem cells ${ }^{16}$. We undeniably need more data, in particular for the identification of MFCs and the mechanisms that support their outgrowth at distant sites.

Christoph A. Klein is at the Division of Oncogenomics, Department of Pathology, University of Regensburg, 93053 Regensburg, Germany. e-mail: christoph.klein@klinik.uni-regensburg.de doi:10.1038/nrc2627-c2

1. Klein, C. A. Parallel progression of primary tumours and metastases. Nature Rev. Cancer 9, 302-312 (2009).

2. Koscielny, S. et al. Breast cancer: relationship between the size of the primary tumour and the probability of metastatic dissemination. Br. J. Cancer 49, 709-715 (1984).

3. Koscielny, S., Tubiana, M. \& Valleron, A. J. A simulation model of the natural history of human breast cancer. Br. J. Cancer 52, 515-524 (1985).

4. Collins, V. P., Loeffler, R. K. \& Tivey, H. Observations on growth rates of human tumors. Am. J. Roentgenol Radium Ther. Nucl. Med. 76, 988-1000 (1956).

5. Engel, J. et al. The process of metastasisation for breast cancer. Eur. J. Cancer 39, 1794-1806 (2003).

6. Friberg, S. \& Mattson, S. On the growth rates of human malignant tumors: implications for medical decision making. J. Surg. Oncol. 65, 284-297 (1997).

Schardt, J. A. et al. Genomic analysis of single cytokeratin-positive cells from bone marrow reveals early mutational events in breast cancer. Cancer Cell 8, 227-239 (2005).

8. Schmidt-Kittler, O et al. From latent disseminated cells to overt metastasis: genetic analysis of systemic breast cancer progression. Proc. Natl Acad. Sci. USA 100, 7737-7742 (2003).

9. Stoecklein, N. H. et al. Direct genetic analysis of single disseminated cancer cells for prediction of outcome and therapy selection in esophageal cancer. Cancer Cell 13, 441-453 (2008)

10. Weckermann, D. et al. Perioperative activation of disseminated tumor cells in bone marrow of patients with prostate cancer. J. Clin. Oncol. 27, 1549-1556 (2009).

11. Stoecklein, N. H. \& Klein, C. A. Genetic disparity between primary tumours, disseminated tumour cells, and manifest metastasis. Int. J. Cancer 126 , 589-598 (2009).

12. Braun, S. et al. A pooled analysis of bone marrow micrometastasis in breast cancer. N. Engl. J. Med. 353, 793-802 (2005).

13. Husemann, Y. et al. Systemic spread is an early step in breast cancer. Cancer Cell 13, 58-68 (2008).

14. Klein, C. A. et al. Genetic heterogeneity of single disseminated tumour cells in minimal residual cancer Lancet 360, 683-689 (2002).

15. Ansieau, S. et al. Induction of EMT by twist proteins as a collateral effect of tumor-promoting inactivation of premature senescence. Cancer Cell 14, 79-89 (2008)

16. Klein, C. A. Cancer. The metastasis cascade. Science 321, 1785-1787 (2008).

Competing interests statement

The author declares no competing financial interests. 